\title{
Study of vesicobullous lesions of the skin clinical and histopathology correlation
}

\author{
Anil Kumar' ${ }^{1}$, Shreya A $\mathbf{S}^{2, *}$, Mirza Asif Baig ${ }^{3}$ \\ ${ }^{\mathbf{1}}$ Associate Professor, ${ }^{\mathbf{2}}$ Assistant Professor, Dept. of Pathology, ESIC Medical College, Gulbarga, Karnataka, ${ }^{3}$ Former Assistant \\ Professor, Dept. of Pathology, Shri B. M. Patil Medical College, Bijapur, Karnataka, India
}

*Corresponding Author:

Email: shreya.shivaraj@yahoo.com

\begin{abstract}
Introduction: Vesiculobullous diseases are a group of disorders in which primary lesion is a vesicle or a bulla on the skin or mucous membrane or both. Though some of the veisculobullous lesions are characteristics in their appearance and distribution, clinically many a times a definitive diagnosis cannot be made by physical examination alone. Then the dermatologists are forced to resort to histopathology for a definite diagnosis and classification. In the present scenario, even though histopathological study is sufficient in most of the cases, it is often accompanied by immunofluorescence antibody tests for confirmation of the diagnosis and monitoring of the disease. Direct immunofluorescence antibody test is a gold standard to confirm the disease and the indirect test helps to monitor the disease.

Objectives: This study was carried out to find out clinical and histopathological correlation in diagnosing the disease.

Materials and Methods: In the present study, histopathological evaluated vesiculobullous lesions over the period of 2 years. A total number of 35 cases were encountered. Staining procedures done are haematoxylin - eosin staining, leishmann staining and direct immunofluorescence stain.

Results: A total number of 35 cases were encountered which constituted $0.70 \%$ of overall biopsy specimen and $12.32 \%$ of skin biopsy specimen. The pemphigus vulgaris formed commonest disease encountered accounting for $34.30 \%$ of all skin lesions. Bullous pemphigoid formed the second most common disease observed (31.34\%) affecting both sexes equally.

Conclusions: All though the 'primary' vesiculobullous lesions of skin are seen in a small group of people, they have been associated with significant a morbidity and mortality. It is important to distinguish each of these entities and separate them appropriate for management and treatment. Even though clinical examination has its own importance, both histopathological and DIF testing is must, as it confirms the diagnosis and subtyping.
\end{abstract}

Keywords: Vesicobullous, Immunofluorescence, Pemphigus, Pemphigoid, Histopathology.

\section{Introduction}

Vesiculobullous diseases are a group of disorders in which primary lesion is a vesicle or a bulla on the skin or mucous membrane or both. Among the various dermatological conditions, vesiculobullous lesions form one of the most frequent clinical problems. It is one of the archetypal reaction pattern of skin to damages caused by both external and internal factors and conditions.

Though some of the veisculobullous lesions are characteristics in their appearance and distribution, clinically many a times a definitive diagnosis cannot be made by physical examination alone. Then the dermatologists are forced to resort to histopathology for a definite diagnosis and classification. Basic histopathological study is helpful in knowing the level of cleavage in the bulla, mechanism of blister formation, the character of inflammatory infilteration, condition of epidermis and dermis lend a possible clue to the etiology and stage of the disease.

Six principal problems encountered while studying the disease;

1. The plane of separation may change as blister ages.

2. Microscopic slit like spaces occur in the epidermis in the category of clefting diseases. Eg: Darier's disease.
3. Evaluation of routine histopathological preparations may not allow one to accurately assess the specific mechanisms of blister formation.

4. The inflammatory cell types infilterating the lesions change as the lesions age.

5. Many of the subepidermal blistering disorders may strikingly mimic each other clinically, leading to inappropriate diagnosis, thus making the histologic descriptions in the literature suspect.

6. Many drugs used either systemically and topically affect the histological picture that may mislead to diagnosis of other conditions. ${ }^{1}$

In the present scenario, even though histopathological study is sufficient in most of the cases, it is often accompanied by immunofluorescence antibody tests for confirmation of the diagnosis and monitoring of the disease. Direct immunofluorescence antibody test is a gold standard to confirm the disease and the indirect test helps to monitor the disease.

Keeping in view of the above facts, it was considered worthwhile to study such disorders histopathologically along with relevant clinical details.

\section{Objectives}

1. To evaluate the pattern of distribution of vesiculobullous skin diseases.

2. To evaluate the histomorphological aspect of diseases. 
3. To study clinical and histopathological correlation in diagnosing the disease.

\section{Materials and Methods}

In the present study, histopathological evaluated vesiculobullous lesions over the period of 6 years in Department of Pathology, ESIC Medical College and Hospital were studied. A total number of 35 cases were encountered.

Skin biopsies for histopathology study were formalin fixed. Staining procedures done are routine haematoxylin - eosin staining methods. Few cases TZANK smears are done, staining method done for these are leishmann staining.

Perilesional skin biopsies, sent in normal saline were used for direct immunofluorescence stain.

\section{Results}

Out of the total 35 cases, 23 cases were of intraepidermal bullous diseases and 11 cases sub-epidermal bullous. One case of dermatitis herpetiformis was seen.

Table 1

\begin{tabular}{|l|c|}
\hline \multicolumn{1}{|c|}{ Disease } & No. of cases \\
\hline PV & 12 \\
\hline PF & 8 \\
\hline P. veg. & 1 \\
\hline PNP & 1 \\
\hline SCPD & 1 \\
\hline Bullous pemphigoid & 11 \\
\hline
\end{tabular}

A total number of 35 cases were encountered which constituted $0.70 \%$ of overall biopsy specimen and $12.32 \%$ of skin biopsy specimen. The pemphigus vulgaris formed commonest disease encountered accounting for $34.30 \%$ of all skin lesions. The peak age incidence for P. vulgaris was $4^{\text {th }}$ and $5^{\text {th }}$ decade with a male female ratio observed being 1:2. Pemphigus foliaceus formed the second common group among epidermal bullous lesions constituting $22.9 \%$ of overall skin biopsy specimens. The commonest age groups in P.foliaceus were $3^{\text {rd }}$ and $4^{\text {th }}$ decades and it was slightly more common in females $(1: 1.67)$.

Bullous pemphigoid formed the second most common disease observed (31.34\%) affecting both sexes equally. One case each of P.vegetans, subcorneal pustular dermatosis, paraneoplastic pemphigus (P.vulgaris type) and dermatitis herpetiformis was observed. P.vegetans was associated with Type II diabetes mellitus. Paraneoplastic pemphigus (P.vulgaris type) was seen in patient with carcinoma of the pyriform fossa (squamous cell carcinoma). Dermatitis herpetiformis was seen in a patient who is intolerant to wheat based food products.

Table 2: Comparison of clinical, histological and DIF

\begin{tabular}{|c|c|c|c|c|c|c|c|c|c|}
\multirow{2}{*}{$\begin{array}{c}\text { Clinical } \\
\text { diagnosis }\end{array}$} & \multirow{2}{*}{ DIF } & \multicolumn{7}{|c|}{ Histological diagnosis } & \multicolumn{2}{c|}{ Total } \\
\cline { 2 - 10 } & & PV & BP & PF & SCPD & P.Veg. & PNP & DH & - \\
\multirow{4}{*}{ PV } & Nil & - & - & - & $1(100.0 \%)$ & - & - & $1(6.7 \%)$ \\
\cline { 2 - 10 } & P & $12(100.0 \%)$ & - & $2(100.0 \%)$ & - & - & - & - & $14(93.3 \%)$ \\
\cline { 2 - 10 } & Total & $12(100.0 \%)$ & - & $2(100.0 \%)$ & $1(100.0 \%)$ & - & - & - & $15(100.0 \%)$ \\
\hline \multirow{3}{*}{ BP } & Nil & - & - & - & - & - & - & $1(100.0 \%)$ & $1(9.1 \%)$ \\
\cline { 2 - 10 } & BP & - & $10(100.0 \%)$ & - & - & - & - & - & $10(90.9 \%)$ \\
\cline { 2 - 10 } & Total & - & $10(100.0 \%)$ & - & - & - & - & $1(100.0 \%)$ & $11(100.0 \%)$ \\
\hline \multirow{2}{*}{ PF } & P & - & - & $6(100.0 \%)$ & - & - & - & - & $6(100.0 \%)$ \\
\cline { 2 - 10 } & Total & - & - & $6(100.0 \%)$ & - & - & - & - & $6(100.0 \%)$ \\
\hline \multirow{2}{*}{ SEBD } & BP & - & $1(100.0 \%)$ & - & - & - & - & - & $1(100.0 \%)$ \\
\cline { 2 - 10 } & Total & - & $1(100.0 \%)$ & - & - & - & - & - & $1(100.0 \%)$ \\
\hline \multirow{2}{*}{ PNP } & PNP & - & - & - & - & - & $1(100.0 \%)$ & $1(100.0 \%)$ \\
\cline { 2 - 10 } & Total & - & - & - & - & - & $1(100.0 \%)$ & - & $1(100.0 \%)$ \\
\hline \multirow{2}{*}{ P. veg. } & P & - & - & - & - & $1(100.0 \%)$ & - & - & $1(100.0 \%)$ \\
\cline { 2 - 10 } & Total & - & - & - & - & $1(100.0 \%)$ & - & - & $1(100.0 \%)$ \\
\hline
\end{tabular}

Table 3: Distribution of various intraepidermal bullous lesions

\begin{tabular}{|l|c|}
\hline \multicolumn{1}{|c|}{ Disease } & No. of cases \\
\hline PV & 12 \\
\hline PF & 8 \\
\hline P. veg. & 1 \\
\hline PNP & 1 \\
\hline SCPD & 1 \\
\hline Total & 23 \\
\hline
\end{tabular}




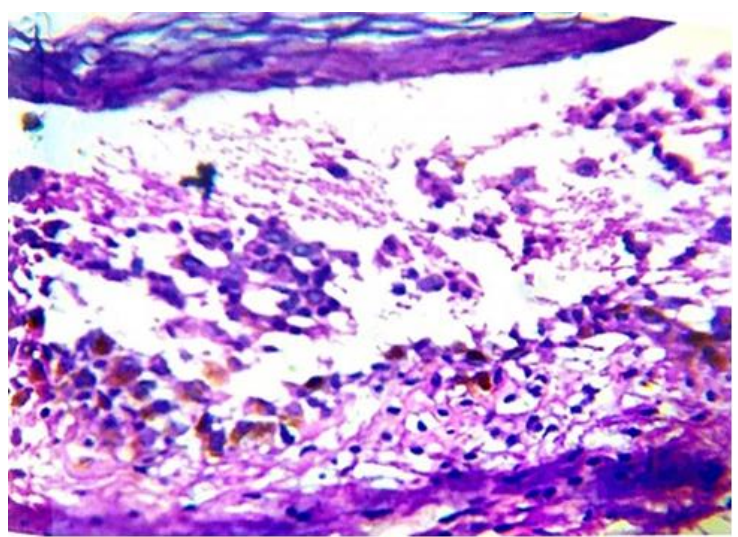

Fig. 1: Neutrophilic iniltration in PNP

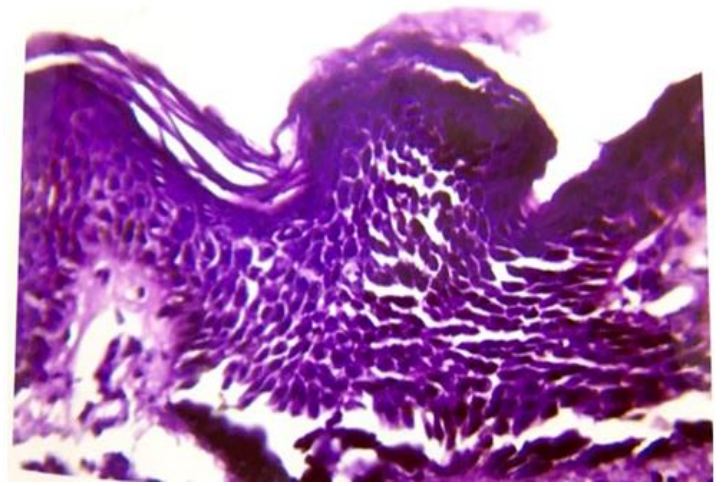

Fig. 2: Spongiosis and suprabasal cleft in early p.vulgaris

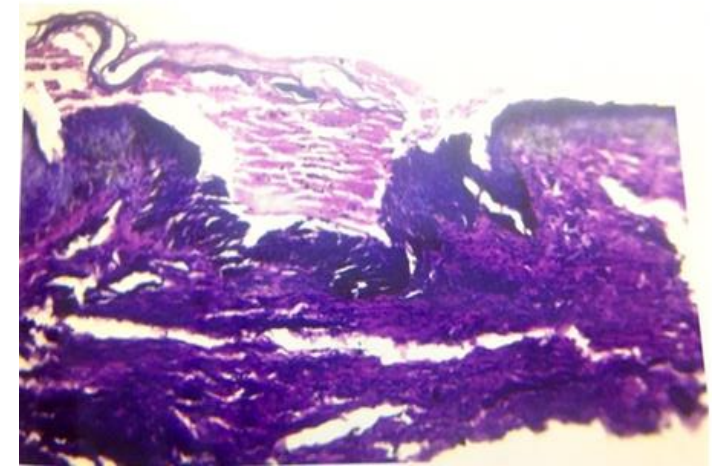

Fig. 3: Subcorneal bulla in p.foliaceus

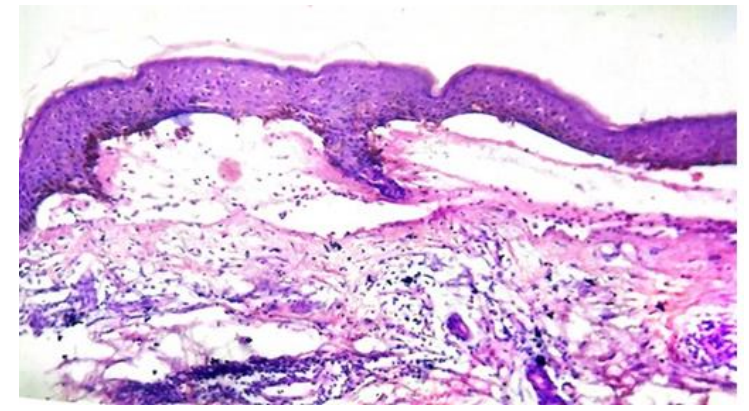

Fig. 4: Subepidermal bulla in b.pemphigoid

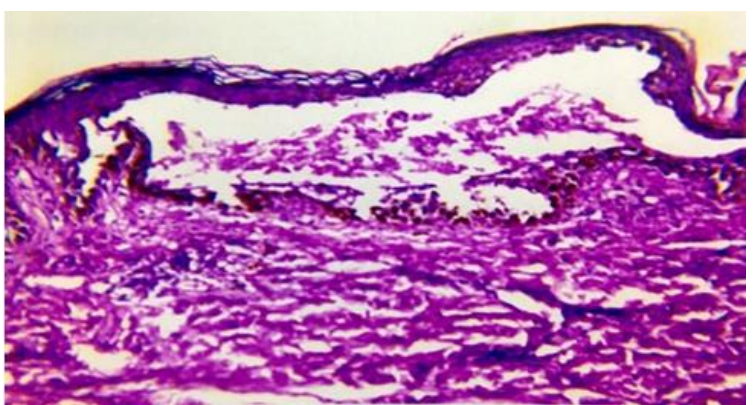

Fig. 5: Suprabasal bulla in p.vulgaris

\section{Discussion}

Pemphigus Vulgaris: Among the vesiculobullous, in the pemphigus group, pemphigus vulgaris is the commonest variety. ${ }^{2}$ Shafi, M et al havestudies 109 cases of pemphigus from Tripoli, Libya and have found that the incidence of pemphigus in Libya is very high, with predominant variant being pemphigys foliaceus. In his study Shafi. M et al also found out that males were predominantly affected than females. ${ }^{3}$ In the present study, pemphigus vulgaris was the most common clinically diagnosed disease accounting for 15 cases, out of which 12 cases were later confirmed by histology and by DIF. There was difference in the diagnosis of 3 cases, out of which 2 cases were pemphigus foliaceus and one case was subcorneal pustular dermatosis.

Even though most common age at presentation is during the $4^{\text {th }}$ decade, we observed that the disease is more common in females (M:F ratio is 1:2) as compared to the western literature where it seen to affect both the sexes equally. The gender differences largely vary from place to place with the environmental and genetic factors playing an important role. ${ }^{4-6}$

Pemphigus Foliaceus: In our study, pemphigus foliaceus consisted of 8 cases accounting for $22.9 \%$ seen in both the sexes equally which is similar to other studies. $^{7-9}$ Out of 8 cases, 6 cases were clinically diagnosed as P.foliaceus and 2 cases as P.vulgaris.

It is important to differentiate between these two, since P.foliaceus has a relatively benign course as compared to P.vulgaris. ${ }^{4,10,11}$

Pemphigus Vegetans: One case of P.vegatans was diagnosed in the study accounting for $4.34 \%$ of pemphigus group of diseases which is similar to the other studies. $^{4,12}$

Histology of the lesion revealed marked acanthosis with eosinophilic abscess within the bulla cavity. Eosoniphilic infiltrate was also noted within the dermis. DIF showed pemphigus type of immunodeposition. The diagnosis of P.vegetans was confirmed but subtyping was done as clinical basis since their histological features are similar in the both Neumann and Hallapeau types. $^{1,9}$

It is important to subtype the disease since Neumann type has similar clinical course as that of P.vulgaris but Hallapeau has more localized, chronic and indolent course and responds well to treatment. ${ }^{9}$ 
Subcorneal Pustular Dermatosis: One case of SCPD was seen in the study. The patient was a 45 year old male who had recurrent papulo vesicular lesions since one and a half years and presented with fresh lesions since 20 days. Patient did not have mucosal lesions.

Histology of lesion revealed subcorneal bulla filled with inflammatory cells predominantly neutrophils and occasional acantholytic cells. Patients hematology report showed neutrophilic leukocsytosis. Histologically a diagnosis of SCPD was made and DIF studies were requested.

DIF with anti IgG antibody test was negative which ruled out pemphigus vulgaris and P.foliaceus. However, a DIF with anti IgA antibody test was advised to rule out a very rare IgA-Pemphigus disease.

Paraneoplastic Pemphigus: A single case of paraneoplastic pemphigus of P.vulgaris type was diagnosed in our study. The patient was a 42 year old male who had carcinoma of pyriform fossa (squamous cell carcinoma). After therapy, he presented with polymorphous bullous lesions over the skin, respiratory and GI systems.

In 2001 Nguyen et al. introduced the concept of paraneoplastic autoimmune multiorgan syndrome (PAMS), highlighting the systemic nature of PNP. ${ }^{13}$ Usually, a neoplasm is detected before the onset of PNP $^{14-16}$ However, PNP is the first clinical manifestation that leads to the detection of an occult tumor in about $30 \%$ of cases. ${ }^{17,18}$ Usually, oral and skin lesions are the earliest manifestations. ${ }^{16,19,20}$

Bullous Pemphigoid: Bullous pemphigoid shows clinical similarity to pemphigus (hence its name) but the blisters are sub-epidermal, not intraepidermal ${ }^{14}$. It is most common in people over the age of 50 years, with male preponderance. ${ }^{15}$ It is a commoner disease in Europe and north America as the average age of the population increases and is characterized by the presence of large, tense bullae, usually on the thighs, arms and abdomen. ${ }^{21}$

In the present study bullous pemphigoid is the second most common disorder which constitutes $31.4 \%$ affecting both sexes equally (M:F ratio 1.2:1). Out of the 11 cases, 10 cases were clinically diagnosed and in one case the disease was provisionally diagnosed as subepidermal bullous disorder - BP/Linear IgA dermatosis under evaluation.

Histologically 9 out of 11 cases had subepidermal bullae and the remaining 2 cases had epidermal bullae with marked re-epithelialisation. Eosinophils rich mixed inflammatory cell infiltration was noted in all the cases.

In all cases, DIF showed linear deposition along dermoepidermal junction.

It is important to know the clinical conditions associated with bullous pemphigoid because:

1. They adversely affect the disease process like $\mathrm{T}_{2} \mathrm{DM}$.
2. Some drugs used in treatment of diseases like betablockers and tab glibeclamide in our study, are known to induce B. pemphigoid in some cases. However, the pathogenesis is still to be established clearly.

3. The bullous disease is by itself known to reactivate and aggravate many conditions as in our study pulmonary tuberculosis was reactivated.

4. Some diseases was totally not related to the disease itself as in our case with lipoma and compound neavus.

Dermatitis Herpetiformis: Olbricht et al in their study of 21 cases have demonstrated papillary microabscesses containing predominantly neutrophils in almost all the cases and they were common in males and the predominant age group was between 20-40 years. ${ }^{22}$ In the present study 1 case of dermatitis herpetiformis is accounted for $2.9 \%$ of all the vesiculobullous lesions studied.

The single patient encountered in this study was a 50 year female who presented with tense bullous lesions over the extensor aspects of limbs, chest and back. Patient was allergic to wheat based products. Clinically the patient was diagnosed to have bullous pemphigoid / dermatitis herpetiformis.

Histologically the biopsy showed a subepidermal bulla with marked mixed inflammatory cell infiltration, re-epithelialisation with the perilesional skin showing elongated papillae but no micro abscesses, which is not pathognomic and is present only in $60 \%$ of the cases.

The DIF with anti IgG antibody is negative. With collective clinical, histological and DIF results a final diagnosis of dermatitis herpetiformis was made.

\section{Conclusion}

All though the 'primary' vesiculobullous lesions of skin are seen in a small group of people, they have been associated with significant a morbidity and mortality. It is important to distinguish each of these entities and separate them appropriate for management and treatment. Even though clinical examination ments its own importance, both histopathological and DIF testing is must, as it confirms the diagnosis and subtyping. Though the full panel of DIF is recommended as a standard procedure combination of DIF with a anti IgGFITC antibody and histopathological study can accurately diagnosis most of the cases as they are economically feasible, seem to be better alternatives.

\section{References}

1. Murphy GF, Sellheyer K and Mihm Mc Jr. Theskin In: Kumar V, Abbas AK, Fausto N, editors. Robbins and Cortan pathologic basis of disease, $7^{\text {th }}$ ed. India: Saunders. 2004;1227-1272.

2. Korman N J. Pemphigus. Journal of American Academy of Dermatologists. 1988;18:1219. 
3. Shafi M, Khatri ML, Mashina ML et al. Pemphigus: A clinical study of 109 cases from Tripoli; Libya. Indian journal of Dermatology Vernerology and Leprology. 1994;60:140-143.

4. Hong WU, Brian S, Terence JH. Non infectious vesiculo bullous and vesciculopustular diseases. In: David EE, George FM, Rosaliets, Bernett LJ Jr, editors. Levers histopathology of the skin, $9^{\text {th }}$ ed. India. Lippincott Willams and Wilkins. 2005; 243-91.

5. Naldi L, Betroni M, Cainelli T and Gruppo Italiano Studi epidermiologici in dermatologia. Feasibility of a registry of pemphigus in Italy: two years experience. Int. $J$ Dermatol. 1993;32:424-7.

6. Nanda A, Dvorak R, Khloud AS, Humocid AS and Alsaleh QA. Spectrum of autoimmune bullous diseases in Kuwait. Int J Dermatol. 2004;43:876-81.

7. Arya SR, Valand AG, Krishna K. A clinicopathological study of 70 cases of pemphigus. Indian J Dermatol Venereol Leprol. 1999;65(4):168-71.

8. Tory PS, George WE and Robert SK. Pemphigus and smoking. Int J Dermatol. 2002;41:528-30.

9. Aboobaker J, Morar N, Ramdial PK and Mickel GH. Pemphigus in South Africa. Int J Dermatol. 2001;40:1159.

10. Huda MM. Afsar MI. A clinicopathological study of pemphigus. I Journ Dermat. 2001;46(2):75-9.

11. Scully C. Pemphigus vulgaris : update on etiopathogenesis, oral manifestations and managements. Crit Rev Oral Biol Med. 2002; 13(5):387-408.

12. Wojnarowska F, Venning VA and Burge SM. Immunobullous diseases In: Burns T, Breathnach Stephen, Cox N ; Griffith SC. editors Rook's textbook of dermatology. $7^{\text {th }}$ ed. Blackwell Science 2005;2: 41.141.2 .

13. Nguyen, V.T.; Ndoye, A.; Bassler, K.D.; Shultz, L.D.; Shields, M.C.; Ruben, B.S.; Webber, R.J.; Pittelkow, M.R.; Lynch, P.J.;Grando, S.A. Classification, clinical manifestations, and im-munopathological mechanisms of the epithelial variant of paraneoplastic autoimmune multiorgan syndrome: A reappraisal of paraneoplastic pemphigus. Arch Dermatol. 2001;137:193-206.
14. Anhalt G.J., Kim S.C., Stanley J.R., Korman N.J., Jabs D.A., Kory M., Izumi H., Ratrie H., Mutasim D., 3rd, Ariss-Abdo L. Paraneoplastic pemphigus. An autoimmune mucocu-taneous disease associated with neoplasia. $N$ Engl J Med. 1990;323:1729-1735. doi: 10.1056/NEJM199012203232503.

15. Vassileva S., Drenovska K., Manuelyan K. Autoimmune blistering dermatoses as systemic diseases. Clin Dermatol. 2014;32:364-375. doi: 10.1016/j.clindermatol.2013.11.003.

16. Zhu X., Zhang B. Paraneoplastic pemphigus. J Dermatol. 2007;34:503-511. doi:10.1111/j.13468138.2007.00322.x.

17. Bialy-Golan A., Brenner S., Anhalt G.J. Paraneoplastic pemphigus: Oral involvement as the sole manifestation. Acta Derm. Venereol. 1996;76:253-254.

18. Lee S.E., Kim S.C. Paraneoplastic pemphigus. Dermatol Sin. 2010;28:1-14. doi: 10.1016/S1027-8117(10)600018 .

19. Beutner EH, Jordan RE, chorzalski TP. The immunopathology of pemphigus and bullous pemphigoid. Journal of Investigations in Dermatology. 1968: 51:6380.

20. Ahmed AR, Hardy D. Bullous permphigoid family of autoimmune diseases. International Journal of Dermatology. 1981;20:541-543.

21. Farmer ER. Subepidermal bullous diseases. Journal of Cutaneous Pathology. 1985;12:316-321.

22. Olbricht SM, Flotte TJ, Collins AB et. Dermatitis Herpetiformis with cutaneous deposition of polyclonal IgA1. Archives of Dermatology. 1986;122:418-421.

How to cite this article: Kumar A, Shreya AS, Baig MA. Study of vesicobullous lesions of the skin clinical and histopathology correlation. J Diagn Pathol Oncol. 2018;4(3):214-218. 